\title{
Gestión de personas el Siglo XXI en el terreno de la educación superior
}

\section{Managing People in the 21st Century in Higher Education}

Ana Domínguez MoRales

ORCID: https://orcid.org/0000-0002-8875-9825

Universidad de Sevilla

Departamento de Derecho del Trabajo y de la Seguridad Social

admorales@us.es

DOI: http://dx.doi.org/10.12795/9788447231003.079

Pp.: 1692-1713 


\section{Descripción del contexto}

El inicio del curso académico 2020/2021, prometía introducirnos en la tan ansiada nueva normalidad, altamente esperada y deseada no solo en el plano familiar, social, económico o laboral, sino también en el educativo.

Vivimos, durante la situación de crisis sanitaria ocasionada por la Covid-19 una situación sin precedentes, también en lo que a la docencia universitaria respecta. Tuvimos que adaptarnos, en cuestión de horas, a un entorno digital para el que no estábamos preparados ni material, ni psicológicamente. No obstante las dificultades, el curso pudo ser finalizado con mayor o menor éxito, pero con un aprendizaje global: las tecnologías de la información en la educación superior han irrumpido con fuerza y vocación de permanencia.

No fue sencillo, tampoco, pensar en los posibles escenarios frente a los que nos enfrentaríamos en el curso 2020-2021. La vuelta a la presencialidad era por todos reclamada, pero con límites en el aforo de estudiantes, salvo en algunas asignaturas de matriculaciones reducidas, como era el caso de los Másteres, en particular, el Máster en Gestión y Desarrollo de Recursos Humanos de la Facultad de Ciencias del Trabajo, cuya asignatura, "Gestión de personas en el Siglo XXI", una asignatura obligatoria del primer cuatrimestre, fue la seleccionada para implantar el presente CIMA.

Como no existía plena seguridad de que el curso académico 2019/2020 pudiera ser impartido de forma presencial - o mejor dicho, semipresencial-, en su totalidad, a pesar de haber apostado por un sistema de asistencia rotatoria llamado a evitar la alta concentración de personas en las aulas, todo el profesorado fue invitado a considerar el denominado "Escenario B". El anterior debía estar

Ciclos de Mejora en el Aula (2020). Experiencias de Innovación Docente de la US Esta obra se distribuye con la licencia Creative Commons 
previsto para una nueva suspensión de la presencialidad y seguimiento de clases online. Entrado el mes de octubre, la previsión se cumplió y toda la comunidad universitaria se ha visto obligada a poner en marcha el plan alternativo, antes indicado.

La asignatura en la que se ha implantado el ciclo de mejora docente es compartida por tres Departamentos de las Facultades de Derecho-Derecho del Trabajo-, Ciencias Empresariales y Económicas- Organización de Empresas- y Psicología-Psicología Social-. El grupo de alumnos estaba formado por 30 alumnos, de los que participaron la gran mayoría en la primera sesión -28-, de carácter presencial, y la totalidad de ellos -30- cuando pasamos a la docencia online. El ámbito temporal del CIMA fue de 8 horas distribuidas en cuatro sesiones que se celebraron los jueves y viernes de 18.30-20.30 h. En principio, la última sesión estaba prevista para el 4 de diciembre. No obstante, la modalidad de docencia online nos permitió adelantarla y así, poder completar el ciclo de mejora de forma previa al momento en que se escriben las presentes líneas.

\section{Diseño del ciclo de mejora para la asignatura Gestión de personas en el S. XXI}

\section{Cuestionario inicial}

La asignatura escogida para implantar el CIMA, corresponde al Máster de Gestión y Recursos Humanos que oferta la Facultad de Ciencias del Trabajo. La misma se imparte, como cada año, por los tres departamentos indicados en el apartado anterior, cada uno de ellos con diferente ámbito de estudio.

Ciclos de Mejora en el Aula (2020). Experiencias de Innovación Docente de la US Esta obra se distribuye con la licencia Creative Commons 
El primer bloque de la misma es precisamente el que yo imparto, destinado a que los alumnos adquieran las competencias necesarias para aprehender cómo se gestiona o se ha de gestionar al personal laboral de las empresas en la actualidad. Para ello, es imprescindible abordar los conceptos básicos relacionados con el Derecho del Trabajo, teniendo en cuenta el escaso grado formativo en la materia de la mayoría de los estudiantes, que provienen de grados ajenos al Derecho. Por ello, resulta idónea, como primera actividad del ciclo de mejora, la realización de un cuestionario previo que ahonda en el conocimiento del estudiantado relativo al modelo de relaciones laborales español, resuelto de forma individual y posteriormente entregado al docente para su examen.

Las preguntas que conforman el cuestionario previo fueron las siguientes:

1. ¿Crees que los condicionantes sociales-económicos-políticos afectan a cómo se gestiona al personal de las empresas? Razona la respuesta.

2. Cita normas que conozcas que regulen las relaciones laborales.

3. ¿Qué ámbito del Derecho del trabajo crees que debe mejorar para gestionar a las personas en el S. XXI?

4. Indica alguna/s diferencias que encuentres entre la gestión del personal hace una décadas y actualmente.

Para evitar respuestas selectivas o poco arriesgadas, se les entregó un cuestionario impreso a cada uno, en el que aparecía un número. De esta forma se conseguía el anonimato del estudiante y se le permitía contestar con mayor libertad y valentía. La idea inicial era volver a repetir la

Ciclos de Mejora en el Aula (2020). Experiencias de Innovación Docente de la US Esta obra se distribuye con la licencia Creative Commons Reconocimiento-NoComercial-SinObraDerivada Internacional (CC BY-NC-ND 4.0.) 
operación al final de las sesiones, lo que se vio, en cierta medida, truncado por el paso a clases online. No obstante, esta dificultad pudo ser superada tal y como se pondrá de manifiesto en el apartado relativo a la secuencia de actividades.

\section{Mapa de contenidos}

El primer bloque temático de la asignatura seleccionada prepara al alumno en las áreas jurídicas laborales que entroncan con la gestión de los recursos humanos a nivel de empresa. Concisamente, se debe abordar el concepto de Derecho del Trabajo, su origen y evolución y posteriormente conocer el modelo de relaciones laborales español en perspectiva constitucional.

Seguidamente se valoran los condicionantes externos al ordenamiento interno que influyen en la configuración de las reglas de gestión de los trabajadores y se vislumbra la contraposición de intereses entre el banco social y el empresarial con los que ha de lidiar el legislador para elaborar normas equitativas y justas, pero tuitivas y garantistas para el trabajador.

Finalmente, nos adentramos en la valoración de dichas normas desde el punto de vista de la eficacia, la efectividad y eficiencia de las mismas, conceptos teóricos examinados desde diferentes disciplinas del conocimiento, que no siempre son aplicados con la misma fortuna o éxito en el desarrollo de las normas que regulan las relaciones laborales.

A continuación se muestra el mapa de contenidos del bloque 1 de la asignatura:

Ciclos de Mejora en el Aula (2020). Experiencias de Innovación Docente de la US Esta obra se distribuye con la licencia Creative Commons 


\section{GESTIÓN DE PERSONAS EN EL S. XXI}

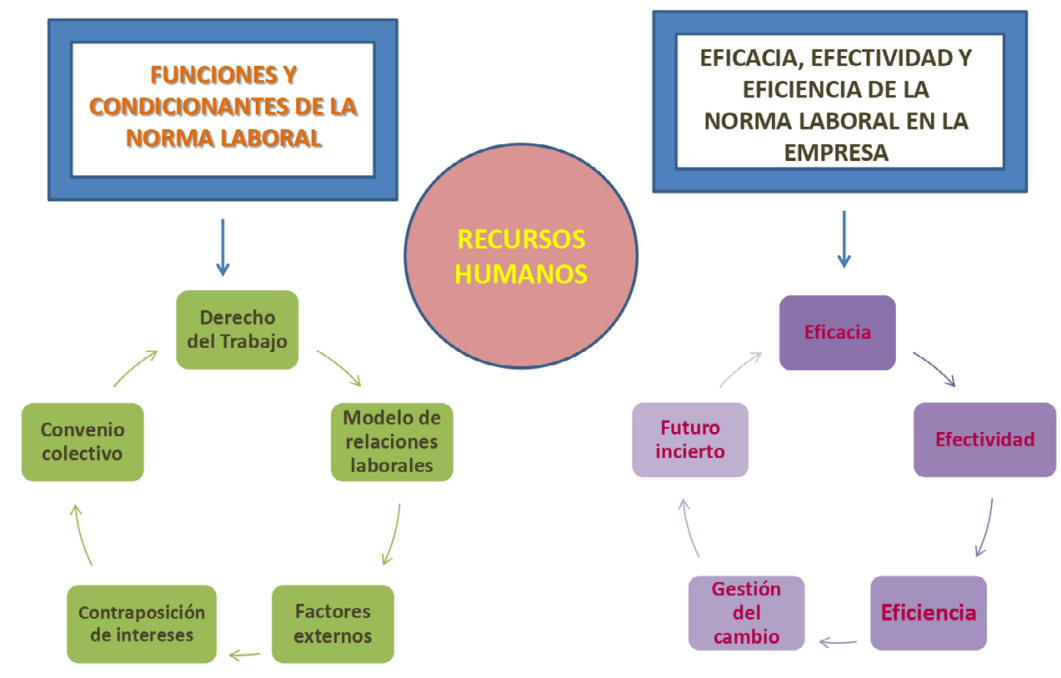

Figura 1. Mapa de contenidos

\section{Modelo metodológico propuesto}

El modelo metodológico utilizado parte de una exploración inicial de conocimientos a través de un cuestionario previo, al que siga un breve debate, para luego intercalar las explicaciones teóricas con supuestos reales. En la siguiente tabla se muestra el modelo adoptado de forma esquemática:
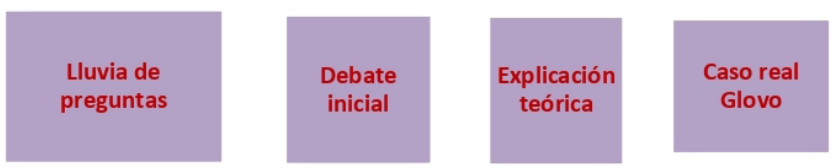

\section{Exposición} en conjunto
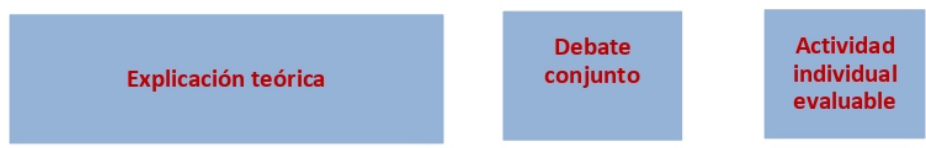

Autoevaluación

Figura 2. Modelo metodológico propuesto

Ciclos de Mejora en el Aula (2020). Experiencias de Innovación Docente de la US 
Con la metodología adoptada y que se puede observar en la Figura 1 se pretende, en primer lugar, asentar las bases del conocimiento mínimo que el alumno debe poseer en relación al Derecho del Trabajo, al modelo constitucional de relaciones laborales y a las vicisitudes a las que se enfrentan empresarios y trabajadores en el mundo globalizado. Todo ello debe ser introducido de forma clara, basada en supuestos fácticos reales, para que los alumnos tengan capacidad de seguir las explicaciones y ofrecer su opinión, en atención al escaso grado formativo en la materia de la mayoría de los estudiantes, que provienen de grados ajenos al Derecho.

Tras un breve debate en el que todos participemos, se pretende intercalar las explicaciones de algunos conceptos básicos, sirviéndose el docente de vídeos didácticos que muestran en pocos minutos el origen y evolución del Derecho del Trabajo en nuestro país.

El caso práctico que sigue, sobre la naturaleza laboral de los prestadores de servicio de transporte de todo tipo de objeto a través de una plataforma digital (Glovo), introduce la problemática que gira entorno a la gestión del personal en el S. XXI, en el que priman, sobre todo, las tecnologías de la información. Este caso no les resulta desconocido, sino más bien al contrario, supone este un servicio que utilizan con frecuencia. Además, tienen noticias de la alta litigiosidad judicial que rodea al mismo gracias a las noticias que pueden leer tanto en telediarios como en sus propias redes sociales.

Este enfoque servirá para que el alumno sea capaz de distinguir los principales modelos de gestión del cambio en el seno de la empresa y, en concreto, en el ámbito de los recursos humanos, habida cuenta de que resulta frecuente que los estudiantes manifiesten, con carácter mayoritario, su intención de pertenecer a dicho sector en el futuro profesional.

Ciclos de Mejora en el Aula (2020). Experiencias de Innovación Docente de la US Esta obra se distribuye con la licencia Creative Commons 


\section{Modelo metodológico propuesto para la docencia no presencial}

La nueva declaración de Estado de alarma y las limitaciones a la movilidad y a la apertura de determinadas actividades no esenciales, vino acompañada del paso de la actividad docente presencial, al modelo online. Siendo así, debimos aplicar el denominado "Escenario B" previsto precisamente para una interrupción radical de la docencia presencial.

En este contexto, nuestro ciclo de mejora docente debió ser adaptado de forma inmediata a la nueva situación justo cuando ya habíamos completado la mitad del mismo. En definitiva, de de las cuatro clases programadas, de dos hora cada una, las dos primeras fueron impartidas de forma presencial, mientras que las otras dos pasaron a celebrarse en formato online, usando para ello la plataforma virtual Blackboard Collaborate Ultra.

En cualquier caso, el cambio no debía alterar el sentido y las secuencias de actividades propuestas.

\section{Secuencia de actividades}

Por las razones antes mencionadas, relativas al desconocimiento generalizado del estudiantado sobre la materia objeto de estudio, se previó comenzar la primera sesión con una encuesta de conocimiento previo, relativo al modelo de relaciones laborales español, que deberán resolver de forma individual que deberían entregar.

La siguiente actividad consistiría en un debate introductorio en el que ellos mismos exterioricen su grado de conocimiento sobre la norma laboral y su impacto en el tejido productivo español y en la gestión del personal laboral.

Ciclos de Mejora en el Aula (2020). Experiencias de Innovación Docente de la US Esta obra se distribuye con la licencia Creative Commons 
Posteriormente, como tercera actividad, se expondría el caso de la empresa Glovo, mediante el visionado del relato de un conductor de la empresa, en el que va describiendo a qué se dedica, cómo ejecuta la prestación y en qué condiciones. Se tratará de resolver la cuestión relativa a si la relación que vincula al rider o repartidor con la empresa citada, se rige la norma laboral ordinaria, o bien, se trata de una relación de trabajo autónoma. Para alcanzar tales conclusiones, se reunirán en equipos y deberán resolver unas preguntas. Las respuestas se verán en común y serán evaluables.

Más adelante, como cuarta actividad, se realizará una clase de introducción teórica sobre los conceptos de eficacia, efectividad y eficiencia de la norma en la gestión del cambio, tratando de propiciar el debate. Posteriormente, como quinta actividad, se les pedirá que, de forma individual, busquen y seleccionen una norma laboral estatal y analicen si la misma resulta eficaz, efectiva y eficiente para gestionar los cambios producidos por las nuevas tecnologías y la globalización mundial, en el seno de las empresas. Esta actividad se realizará en clase y también será evaluable.

Por último, como sexta actividad, se volverá a pasar el cuestionario inicial para poder evaluar la progresión del aprendizaje del alumno a lo largo de las sesiones.

\section{Evaluación}

La evaluación del aprendizaje de los alumnos estaría integrada, por un lado, por el grado de participación e involucración de los mismos en las actividades propuestas. Lo más importante, desde mi punto de vista, es que los alumnos sean capaces de comunicarse con sus compañeros y con el docente, ordenar y expresar sus ideas y 
poder aceptar o admitir la de otros compañeros, quizás más acertadas. En el área de recursos humanos, el trabajo en equipo y colaborativo supone una seña de identidad.

Se valora que el alumno atienda, participe en debates generales y grupales, así como en la realización de las actividades que se van sucediendo. En caso de no asistir a clase, ni participar, ni entregar ninguna actividad por escrito, la última de las previstas, esto es, la evaluación de una norma interna o internacional desde la perspectiva de la eficacia, efectividad y eficiencia de la misma servirá de calificación única y total de este bloque de la asignatura.

\section{Aplicación del Ciclo de Mejora en el Aula}

\section{Desarrollo secuencial}

Antes de comenzar a describir las actividades que se llevaron a cabo, se debe reseñar un cambio en la organización de las sesiones motivada por el paso de la docencia presencial al formato online. Si bien estaba previsto impartir tres sesiones consecutivas y una última separada de las anteriores (por cuestiones internas de organización de la asignatura), finalmente se decidió que cada profesor realizara un bloque de cuatro sesiones consecutivas.

A pesar del cambio de modalidad de impartición de clases, pasando estas al formato online, las dos últimas sesiones se siguieron con regularidad, sin alterar ni el fondo ni la secuencia de actividades determinadas en el diseño previo del CIMA, pero de forma remota.

Como antes advertimos, por razones de falta de conocimiento generalizado del estudiantado sobre la materia objeto de estudio, resultaba idóneo comenzar la primera 
sesión con una encuesta de conocimiento previo, relativo al modelo de relaciones laborales español, que debieron resolver de forma individual y posteriormente entregar a la profesora.

Al hilo de la actividad anterior, en la que los estudiantes debían responder a preguntas dirigidas a valorar qué condicionantes económicos, políticos, sociales o culturales afectaban a la producción del Derecho y, en particular, del Derecho del Trabajo, tanto en la época actual como en tiempos pasados, se proyectó un video de contenido histórico en el que se evidenciaba el origen del Derecho del Trabajo motivado por el movimiento obrero de finales del S. XIX. Finalizada su proyección, se inició un debate grupal en el que los estudiantes exteriorizaron su grado de conocimiento sobre las normas laborales -cómo y por qué se aprueban- y su impacto en el tejido productivo español y en la gestión del personal laboral.

Centrados ya en la materia objeto de aprendizaje, se siguió con explicaciones que fueron complementadas con otro vídeo de contenido cómico-irónico, en el que registraba una entrevista de trabajo realizada por la jefa de selección de recursos humanos de una empresa a un candidato preseleccionado. La entrevistadora, tras alabar el extenso y brillante currículum de quien se postulaba al puesto vacante, ponía a su disposición un contrato de trabajo con un salario irrisorio, que acabó aceptando el entrevistado.

Tras ello, la conversación avanzó, momento en el que la entrevistadora pudo entrever las faltas de uso del lenguaje que cometía el ya nuevo trabajador, y la escasez de conocimientos especializados en la materia objeto del contrato de trabajo que poseía, a pesar de los numerosos títulos académicos del mismo. En definitiva, la grabación no reflejaba más que una crítica al sistema actual de relaciones laborales, que basa en un amplio porcentaje de 
casos la contratación del personal en aspectos objetivos más que en las propias capacidades y competencias de las personas. Esto sirvió, en definitiva, para que los alumnos pudieran observar que las reglas de aplicación práctica del Derecho del Trabajo no siempre van de la mano con el contenido teórico de las normas que lo regulan.

En la siguiente sesión se comenzó con la visualización de obras de arte de principios del S. XX, ilustrativas del movimiento obrero español. Se pedía a los alumnos identificar el sentido de las mismas así como evaluar las semejanzas y diferencias entre las reclamaciones de los trabajadores 100 años atrás y las que se dan en la actualidad.

A través de los cuadros seleccionados, de los que se podían deducir manifestaciones de trabajadores que portaban carteles con consignas referidas al aumento de salario, de estabilidad o de protección de la seguridad y salud en las empresas, se pone en evidencia cómo las principales divergencias entre el conflicto social del hace más de un siglo y el actual se sitúa más en el terreno de lo formal, coincidiendo en muchas ocasiones las propuestas de mejora de tipo material. Correlativo a lo anterior, se avanzó en la materia con explicaciones de la profesora acerca del modelo de relaciones laborales español, basado en la regulación que prefigura la Constitución Española y que configura la contraposición de intereses inherente a las relaciones laborales entre empresarios y trabajadores, así como formula vías para garantizar la protección del trabajador frente a la superioridad de poder del empresario, tanto en su vertiente individual como en la colectiva.

La tercera sesión tuvo como inicio la proyección de un video que retrataba las vivencias, el día a día, de un repartidor de comida a domicilio contratado por la plataforma digital Glovo. El repartidor va describiendo a qué se dedica, cómo ejecuta la prestación y en qué condiciones. Posteriormente, analizamos, en un foro común, si la relación jurídica que vincula al prestador de servicios con Glovo se 
ajusta a las características de laboralidad contenidas en el art. 1.1 del Estatuto de los Trabajadores, o por el contrario se excluye de su ámbito de aplicación. En tal caso, la vinculación entre ambos sujetos podría ser de tipo mercantil o civil, en cuyo caso el repartidor solo alcanzaría a ser reconocido como trabajador autónomo.

Los alumnos tuvieron oportunidad de explorar jurisprudencia y doctrina sobre este asunto durante unos minutos antes de iniciar el debate. Se daba la circunstancia el Tribunal Supremo en una reciente sentencia se pronunció sobre el particular, admitiendo la existencia de laboralidad en la prestación de servicios de un repartidor de Glovo (STS de 25 de septiembre 2020).

Para alcanzar las conclusiones exigidas por la profesora, se reunieron en equipos y resolvieron las preguntas que les fue planteando. Las respuestas se pusieron en común y fueron posteriormente evaluadas, no por su acierto, sino por la profundidad y coherencia en la argumentación.

Finalmente, en la última sesión, se comenzó con una explicación introductoria sobre los conceptos de eficacia, efectividad y eficiencia de la norma laboral en la gestión del cambio, tratando de propiciar el debate sobre los conceptos explicados, que no resultaron ser de fácil asimilación por los alumnos.

Mientras que el primero de ellos tiene que ver con la aptitud de la norma para alcanzar los fines u objetivos por ella previstos, la efectividad evidencia el grado de cumplimiento por parte de sus destinatarios. Finalmente, la eficiencia responde a la relación de coste/beneficio de lo previsto en una ley determinada, esto es, entre el mecanismo previsto para alcanzar un fin y la utilidad o provecho alcanzado en la aplicación de la norma.

Posteriormente, como penúltima actividad, se les pidió que, de forma individual, buscaran y seleccionaran una 
norma laboral estatal o internacional. Podían utilizar tanto un solo precepto como una ley completa. Dicha norma o precepto debía ser evaluado a la luz de los conceptos de eficacia, efectividad y eficiencia de las normas desde su punto de vista, implementando en sus respuestas todo lo aprehendido durante las sesiones anteriores. Además, debían pronunciarse acerca de si la misma resultaba apta para gestionar los cambios producidos por las nuevas tecnologías y la globalización mundial, en el seno de las empresas. Esta actividad también será evaluable.

Por último, aprovechando uso de las tecnologías y las sesiones online, se les pidió que de forma individual y fuera del horario lectivo, volvieran a responder al cuestionario inicial de nuevo de forma anónima. Esto se conseguiría porque indicarían en el documento enviado el número que les había sido asignado la primera vez que respondieron al mismo. De esta forma, conseguiría evaluar de forma objetiva, la progresión del aprendizaje del alumno a lo largo de las sesiones.

\section{Evaluación del aprendizaje de los estudiantes}

La evolución del aprendizaje de los estudiantes se ha evaluado mediante la comparación de las respuestas obtenidas del cuestionario inicial y final mediante escaleras de conocimiento

De un total de 30 matriculados, solo asistieron 28 en las dos primeras sesiones, por lo que el cuestionario inicial y final solo ha sido realizado por los que asistieron a las 4 sesiones inicialmente programadas. No se realizó la encuesta a los dos alumnos que no participaron en las primeras sesiones, porque a pesar de no acudir a la cita presencial, tenían a su disposición los materiales desde el día antes de la primera sesión, que fueron colgados por la profesora en enseñanza virtual, y de ahí que no se pudiera garantizar el efecto deseado de la encuesta, dirigido a valorar el conocimiento previo a la impartición de la docencia correspondiente y el obtenido una vez finalizada esta.

Ciclos de Mejora en el Aula (2020). Experiencias de Innovación Docente de la US Esta obra se distribuye con la licencia Creative Commons 
- Primera pregunta: ¿Crees que los condicionantes sociales-económicos-políticos afectan a cómo se gestiona al personal de las empresas? Razona la respuesta

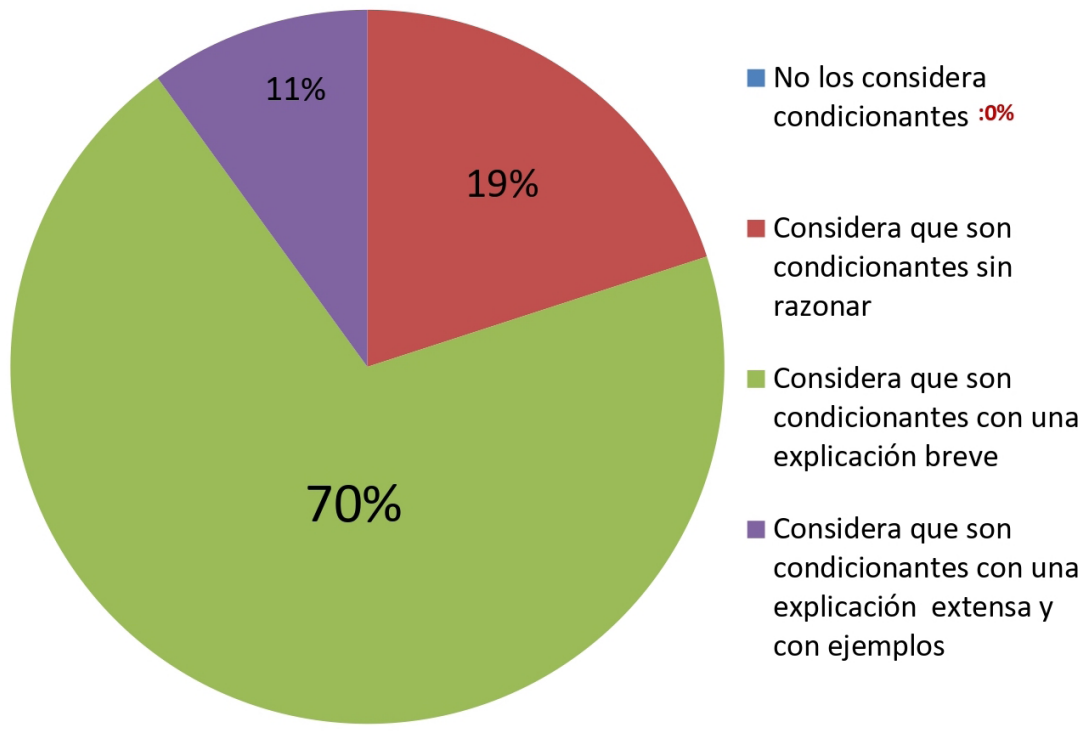

Figura 3. Pregunta 1 Cuestionario inicial.

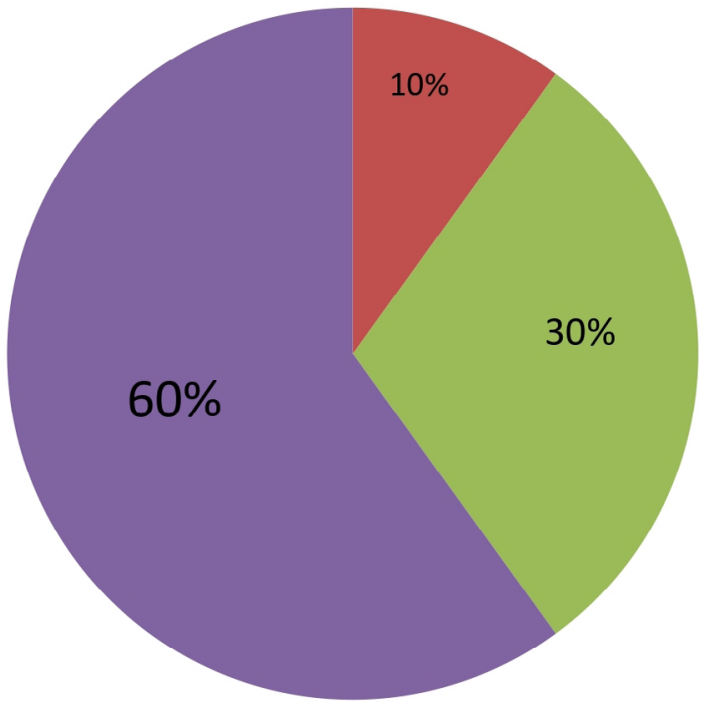

- No los considera condicionantes $(0 \%)$

Considera que son condicionantes sin razonar

- Considera que son condicionantes con una explicación breve

- Considera que son condicionantes con una explicación extensa y con ejemplos

Figura 4. Pregunta 1 Cuestionario final.

Ciclos de Mejora en el Aula (2020). Experiencias de Innovación Docente de la US 
- Segunda pregunta: Cita normas que conozcas que regulen las relaciones laborales.

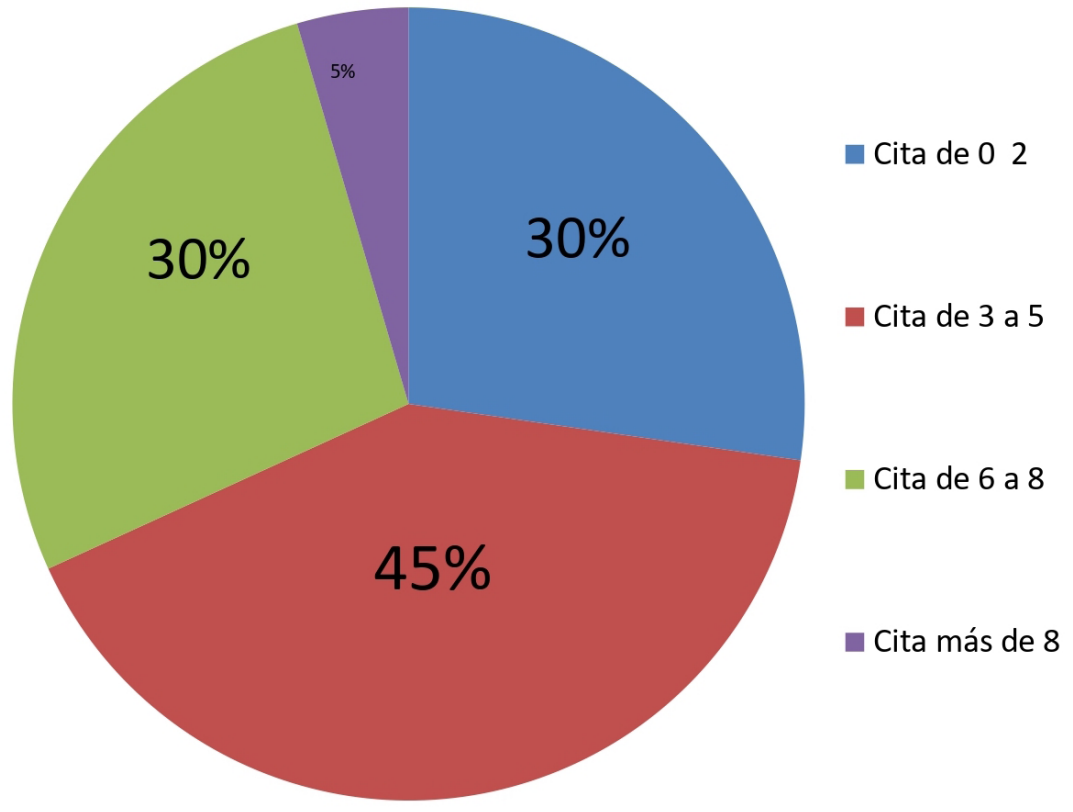

Figura 5. Pregunta 1. Cuestionario inicial

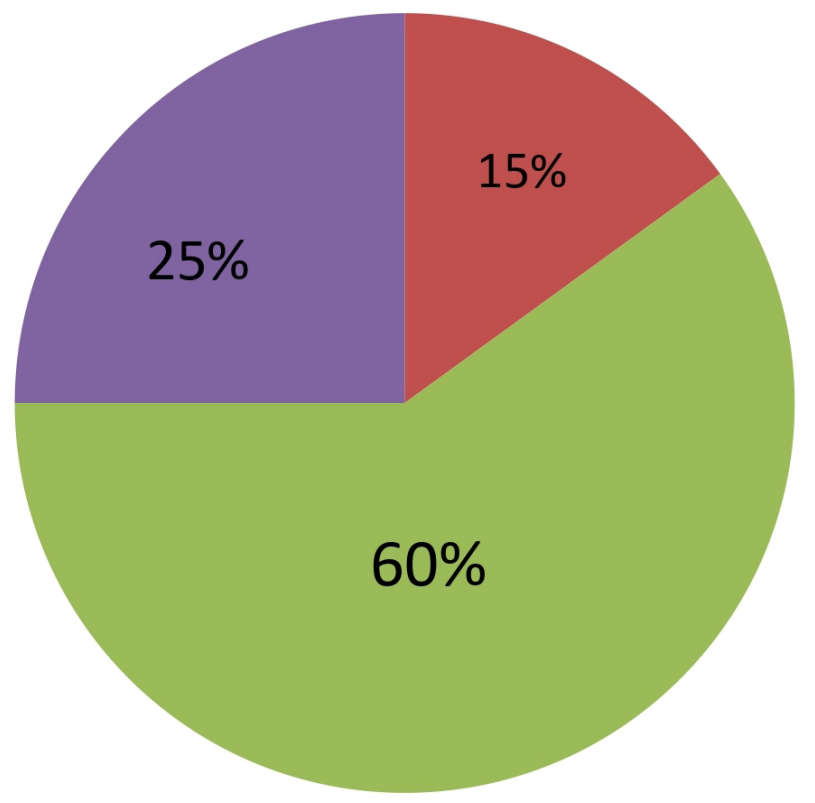

- Cita de $02:(0 \%)$

- Cita de 3 a 5

Cita de 6 a 8

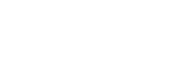

- Cita más de 8

Figura 6. Pregunta 2. Cuestionario final

Ciclos de Mejora en el Aula (2020). Experiencias de Innovación Docente de la US (c) (i) $\odot$ Esta obra se distribuye con la licencia Creative Commons Internacional (CC BY-NC-ND 4.0.) 
- Tercera pregunta: ¿Qué ámbito del Derecho del trabajo crees que debe mejorar para gestionar a las personas en el S. XXI?

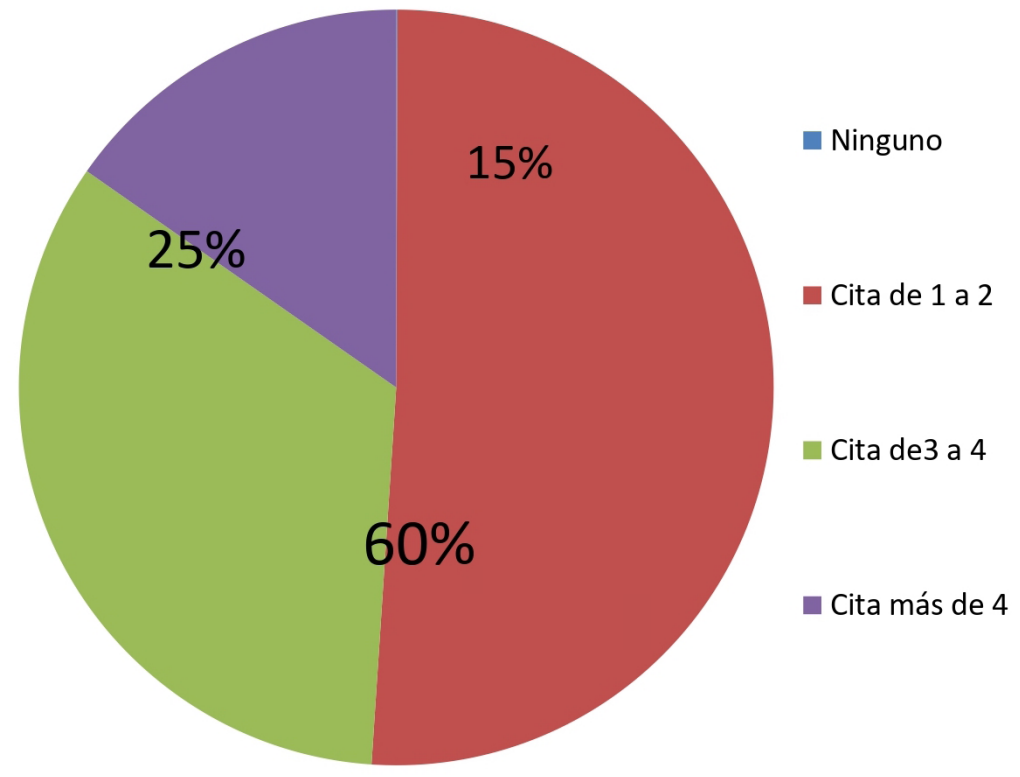

Figura 7. Pregunta 3. Cuestionario inicial

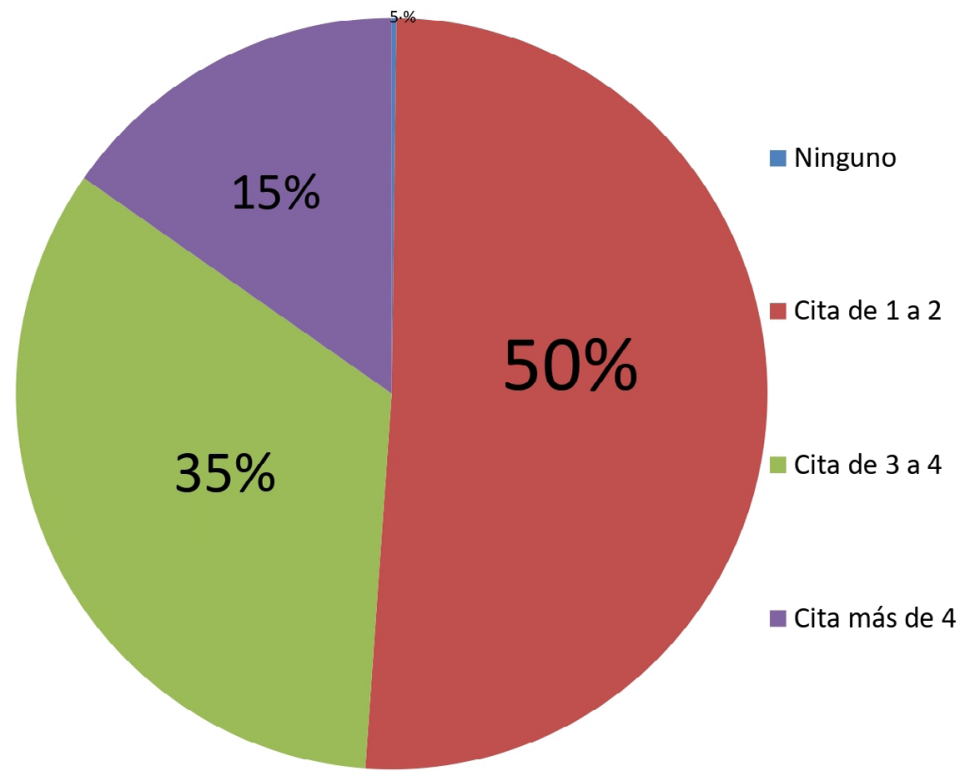

Figura 8. Pregunta. Cuestionario final

Ciclos de Mejora en el Aula (2020). Experiencias de Innovación Docente de la US (c) (i) $\odot$ Esta obra se distribuye con la licencia Creative Commons Reconocimiento-NoComercial-SinObraDerivada 
- Cuarta pregunta: Indica alguna/s diferencias que encuentres entre la gestión del personal hace una décadas y actualmente.

a) Cuestionario inicial

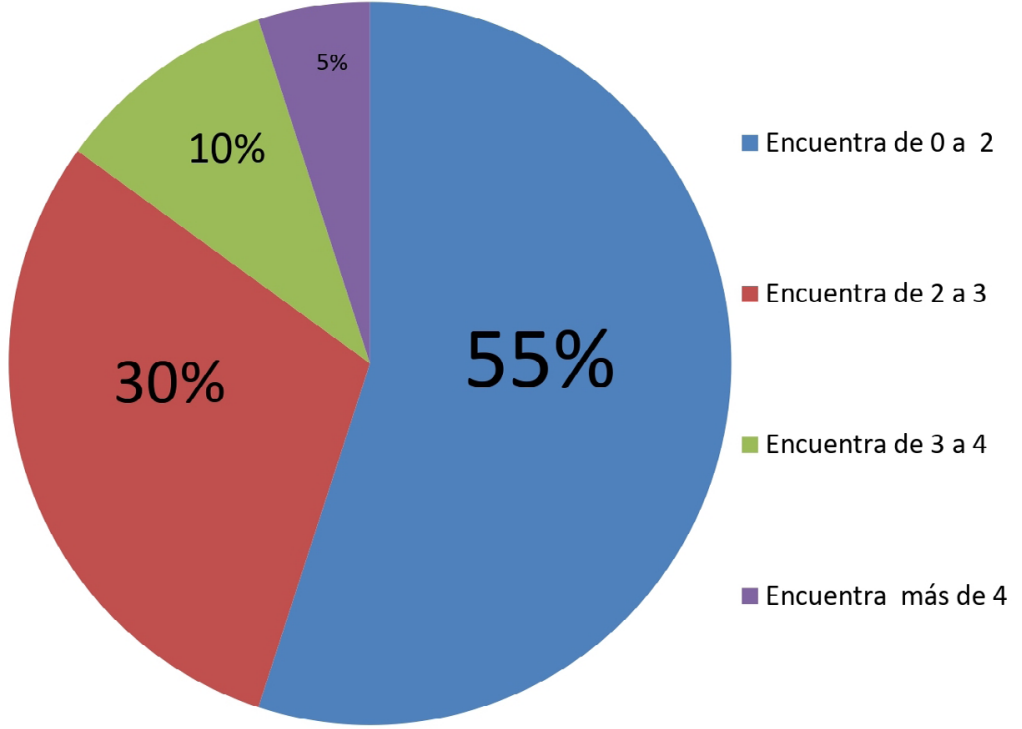

Figura 9. Pregunta 4. Cuestionario inicial

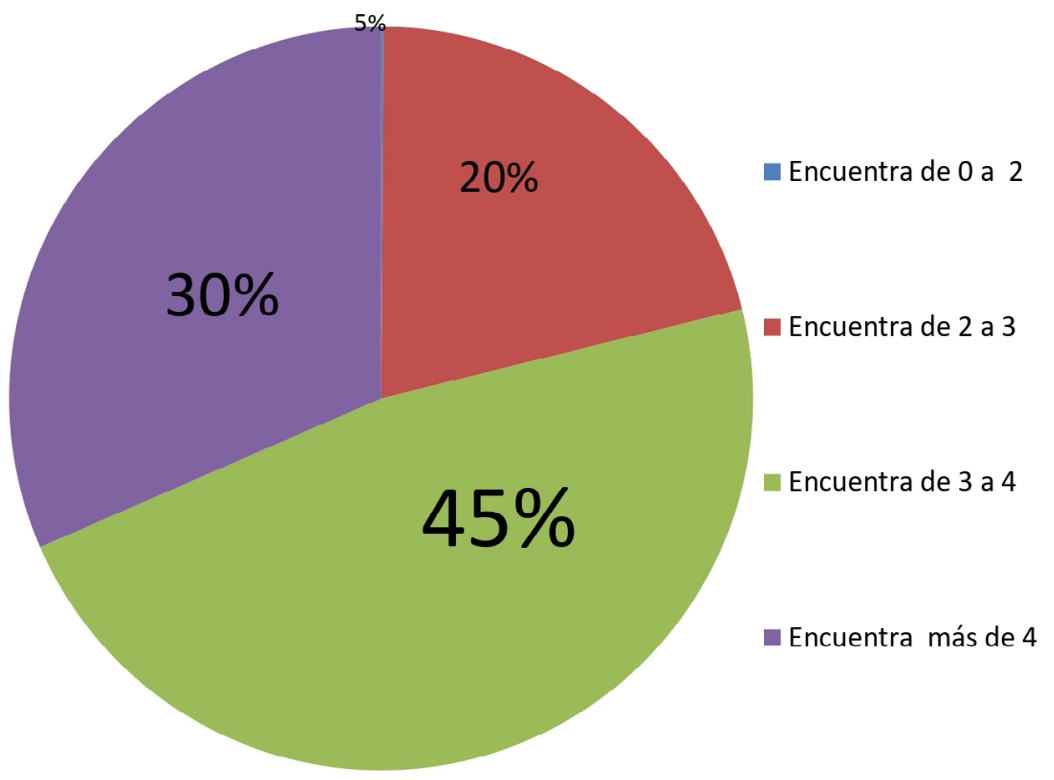

Figura 10. Pregunta 4. Cuestionario final

Ciclos de Mejora en el Aula (2020). Experiencias de Innovación Docente de la US (c) (i) $\odot$ Esta obra se distribuye con la licencia Creative Commons Reconocimiento-NoComercial-SinObraDerivada $\quad 4.0$ Internacional (CC BY-NC-ND 4.0.) 


\section{Autoevaluación del Ciclo de Mejora en el Aula}

El resultado de la aplicación del ciclo de mejora docente es manifiestamente positivo por varias razones.

La primera de ellas es que se ha podido comprobar una mejora en el aprendizaje del alumno, a pesar de que se ha variado ligeramente la metodología de la enseñanza previamente utilizada por la modificación del sistema de impartición de docencia de un modelo presencial a otro online.

Las escaleras de conocimiento precedentes muestran mejoras en todas las preguntas realizadas. En algunos casos, la subida de peldaños porcentuales desde los inferiores hasta los superiores resulta menos evidente, como por ejemplo, en la pregunta 3 donde se les pedía a los estudiantes su opinión sobre qué ámbito del Derecho del trabajo consideraban que debía mejorar para gestionar a las personas en el S. XXI. Mientras que en el escalón cuarto, en el que se situaban los que daban más de 3 zonas de manifiesta necesidad de mejora, se encontraba el $15 \%$, en el cuestionario final aumentó solo en un 5\% el número de alumnos que aportaban tal información cualificada.

En otros casos la evolución se advierte de forma más evidente. Esto sucede, a modo de ejemplo, en la pregunta cuarta, en la que se pretendía que el alumno indicara alguna/s diferencias entre la gestión del personal hace una décadas y actualmente. Mientras que en el primer escalón de conocimiento (de 0 a 2) se encontraba más de la mitad de la clase (55\% de los asistentes), el cuestionario final muestra que tan solo una mínima proporción (5\%) sigue manteniendo esa misma opinión, mientras que aumenta el porcentaje de alumnos que encuentra más de 3 diferencias en la gestión del personal en la actualidad en comparación con una década atrás (el 65\%, distribuidos entre los escalones tercero y cuarto).

Ciclos de Mejora en el Aula (2020). Experiencias de Innovación Docente de la US Esta obra se distribuye con la licencia Creative Commons 
Haciendo una evaluación global del cima, debemos destacar la puesta en práctica de algunos principios didácticos que guiaron la elaboración y aplicación del mismo. El primero de ellos encuentra al estudiante como el epicentro del conocimiento autónomo, encargándose al mismo la tarea de construir dicho conocimiento a medida en que desarrolla las actividades prácticas que le permiten desarrollar sus propias aptitudes y retener mayor información que atendiendo a una clase teórica o magistral (Finkel, 2008).

Por otro lado, se pone de manifiesto que el estudiante retiene mayor información cuando resulta estimulado y no sobresaturado de información que probablemente acabará olvidando, habiendo sido demostrado que el aprendizaje funcional centrado en casos prácticos reales y con proyección más allá de las propias aulas, resulta más adecuado a los fines de la enseñanza (Bain, 2007).

Finalmente, se debe destacar el éxito en la implantación de este CIMA aun habiendo experimentado el cambio de enseñanza presencial a enseñanza online justo en el ecuador del mismo, sin ningún inconveniente destacable, sino antes al contrario, permitiendo que el $100 \%$ de los alumnos matriculados pudieran asistir y participar en las sesiones que se impartieron en modalidad telemática. No obstante, se pudo observar que parte del alumnado no fue tan participativo en las clases online como que en las telemáticas, quizás debido al hecho de que no se le obligaba ni a encender la cámara ni el micrófono si no querían participar de forma activa, eliminando cualquier posibilidad de que el profesor pudiera aseverar que el alumno seguía la sesión.

Ciclos de Mejora en el Aula (2020). Experiencias de Innovación Docente de la US Esta obra se distribuye con la licencia Creative Commons 
En suma, la aplicación de modelos de enseñanza que priorizan el trabajo del alumno y centralizan en él la preparación y organización de cualquier asignatura, se alza como un sistema más eficiente, no ya disruptivo sino cada vez más extendido y que bien compagina con el uso destacado de las tecnologías de la información en los entornos docentes en situaciones no coyunturales, sino estructurales.

Palabras clave: Derecho del Trabajo, modelo de relaciones laborales, gestión de personas, docencia universitaria online, aprendizaje funcional, innovación docente.

Key words: Labour Law, labour relations framework, managing people, online university teaching, functional learning, educational innovation.

Ciclos de Mejora en el Aula (2020). Experiencias de Innovación Docente de la US Esta obra se distribuye con la licencia Creative Commons 


\section{Bibliografia}

Bain, K. (2007). Lo que hacen los mejores profesores universitarios. Valencia: Servicio de Publicaciones de la Universidad de Valencia.

Cruz Villalón, J. Compendio de derecho del trabajo 13a ED (2020). Valencia: Tirant Lo Blanch.

Finkel, D. (2008) Dar clase con la boca cerrada. Valencia: Servicio de Publicaciones de la Universidad de Valencia.

Herrero-Vázquez, E.A. y Torres-Gordillo, J.J., (2020). "ECO en la educación superior: un aprendizaje resonante". En M. Reyes-Tejedor, D. Cobos-Sanchíz y E. López-Meneses (Coords.), Innovación pedagógica universitaria: reflexiones y estrategias (cap. 8). Barcelona: Octaedro. $<$ _cap.8.edu.juanj.innovacion.final.pdf>.

Melero-Aguilar, N. Torres-Gordillo, J.J. y García-jiménez, J. (2020). Retos del profesorado universitario en el proceso de enseñanza-aprendizaje: aportaciones del método ECO (Explorar, Crear y Ofrecer). Formación Universitaria, 13(3), 157-168. doi:10.4067/S0718-50062020000300157

Mora, F. (2017). Neuroeducación: Sólo se puede aprender aquello que se ama. Madrid: Alianza Editorial.

Fernández Enguita, M. (2018). El aula está muy lejos de la marcha del cambio social, del mundo real entrevista). Recuperado de: https://www.diariodesevilla.es/ entrevistas.

Rodríguez Piñero-Royo, M. (2012). La enseñanza del Derecho del Trabajo en los Grados de estudios laborales. En J. Cruz Villalón, J. y M. Rodríguez-Piñero Royo, La enseñanza del Derecho del Trabajo y de la Seguridad Social en los nuevos Planes de Estudio. Murcia: Laborum.

Torres-Gordillo J.J., García-Jiménez, J. \& Herrero-Vázquez, E.A. (2020). Contributions of technology to cooperative work for university innovation with Design Thinking". Pixel-Bit. Revista de Medios y Educación, 59, 27-64. doi:10.12795/ pixelbit.74554

Torres-Gordillo, J.J. Melero-Aguilar, N. \& García-jiménez, J. (2020). "Improving the university teaching-learning process with ECO methodology: Teachers' perceptions". Plos One, 15(8), 1-14. e0237712. doi:10.1371/journal.pone.0237712

Ciclos de Mejora en el Aula (2020). Experiencias de Innovación Docente de la US Esta obra se distribuye con la licencia Creative Commons 